\title{
Self-frequency-doubling of ultrafast laser inscribed neodymium doped yttrium aluminum borate waveguides
}

\author{
Ningning Dong, ${ }^{1}$ J. Martínez de Mendivil, ${ }^{2}$ E. Cantelar, ${ }^{2}$ G. Lifante, ${ }^{2}$ \\ J. Vázquez de Aldana, ${ }^{3}$ G. A. Torchia, ${ }^{4}$ Feng Chen, ${ }^{1, b)}$ and Daniel Jaque ${ }^{2, a)}$ \\ ${ }^{1}$ School of Physics, State Key Laboratory of Crystal Materials, Shandong University, Jinan 250100, \\ People's Republic of China \\ ${ }^{2}$ Departamento de Física de Materiales, Facultad de Ciencias, Universidad Autónoma de Madrid, \\ Madrid 28049, Spain \\ ${ }^{3}$ Departamento Física Aplicada, Facultad Ciencias Físicas, Universidad de Salamanca, \\ Salamanca 37008, Spain \\ ${ }^{4}$ CIOp, Centro de Investigaciones Ópticas, CIC-Conicet, CC 124, La Plata 1900, Argentina
}

(Received 16 March 2011; accepted 11 April 2011; published online 3 May 2011)

\begin{abstract}
Buried channel optical waveguides, supporting orthogonal polarizations, have been fabricated in a neodymium doped yttrium aluminum borate nonlinear laser crystal by ultrafast laser inscription following the so-called "double line" approach. Confocal fluorescence and second harmonic imaging experiments have revealed that the original fluorescence and nonlinear properties have been not deteriorated by the waveguide inscription procedure. Preliminary laser experiments have shown the ability of the fabricated structures for green laser light generation under $808 \mathrm{~nm}$ optical pumping by self-frequency-doubling of the $1.06 \mu \mathrm{m}$ laser line of neodymium ions. (C) 2011 American Institute of Physics. [doi:10.1063/1.3584852]
\end{abstract}

Among the multifunctional optical systems currently proposed as building-blocks of integrated devices, selffrequency-converted (SFC) laser crystals are one of the most widely studied. SFC systems are constituted by a nonlinear crystal which is optically activated by laser ions (usually rare earth ions such as $\mathrm{Nd}^{3+}$ or $\left.\mathrm{Yb}^{3+}\right) .{ }^{1}$ Under laser operation the nonlinear properties of the host matrix activate frequencymixing processes between the different intracavity laser radiations that propagate along the gain medium (pump and laser). These intracavity nonlinear processes lead to the generation of new laser frequencies without the requirement of additional intracavity optical components, i.e., allowing for highly compact multiwavelength laser sources. ${ }^{1}$ Among the different SFC crystals reported up to now, neodymium doped yttrium aluminum borate (hereafter $\mathrm{Nd}: \mathrm{YAB}$ ) is, without doubts, a paradigm. ${ }^{2}$ It combines the good thermal, mechani$\mathrm{cal}$, and nonlinear properties of the YAB host with the outstanding fluorescence properties of Nd ions. ${ }^{3,4}$ Based on this unique combination, multifrequency laser light oscillation in the three fundamental colors (red, green, and blue) has been demonstrated from a unique $\mathrm{Nd}: \mathrm{YAB}$ element operating at $1.3 \mu \mathrm{m} .{ }^{5}$ In its simplest version, involving only the second harmonic (SH) of the $1064 \mathrm{~nm}$ laser line of $\mathrm{Nd}^{3+}$ ions, pumpto-green optical conversion efficiencies as high as $25 \%$ have been reported. ${ }^{6}$ Further incorporation of Nd:YAB SFC lasers in miniaturized optical devices requires the controlled fabrication of optical waveguides (WGs) in the bulk material. Up to now, WGs in Nd:YAB crystals have been only reported by light ion implantation, without demonstrating any selffrequency-doubling (SFD) laser ability. ${ }^{7}$ In order to achieve this goal the fabricated structures should satisfy following three main requirements: (i) the fabrication procedure should preserve the original fluorescence and nonlinear properties of the Nd:YAB system, (ii) the fabricated WGs should support

\footnotetext{
a) Electronic mail: daniel.jaque@uam.es.

${ }^{b)}$ Electronic mail: drfchen@sdu.edu.cn.
}

both TE and TM modes so that birefringence-based phase matching (PM, requiring orthogonal polarizations for fundamental and second harmonic waves) will be possible, and (iii) light confinement should be achieved in a large spectral range so that spatial confinement of both fundamental and second order radiation would be achieved.

In this letter, we report on the fabrication of optical WGs in a Nd:YAB crystal orientated along the $1064 \mathrm{~nm}$ $\rightarrow 532 \mathrm{~nm}$ PM direction by ultrafast laser inscription following the so-called "double-line" approach. We have experimentally determined the writing parameters leading to infrared and visible optical confinement for both TM and TE polarizations while preserving the original fluorescence and nonlinear properties of the Nd:YAB system. The potential application of the fabricated structures as SFD laser WGs has been demonstrated.

The Nd:YAB crystal used in this work was a $2 \mathrm{~mm}$ $\times 2 \mathrm{~mm} \times 5 \mathrm{~mm}$ prism with its longest dimension nominally orientated for Type I $1064 \mathrm{~nm} \rightarrow 532 \mathrm{~nm}$ birefringence PM. The $\mathrm{Nd}^{3+}$ concentration was 5 at. \% leading to an absorption coefficient of $5 \mathrm{~cm}^{-1}$ at $808 \mathrm{~nm}$. In addition to the two $2 \mathrm{~mm} \times 2 \mathrm{~mm}$ end faces used for laser experiments, one of the $2 \mathrm{~mm} \times 5 \mathrm{~mm}$ faces was also polished to optical quality in order to allow laser inscription along the longest dimension. For WG fabrication we used an amplified Ti:sapphire femtosecond (fs) laser operating at wavelength of 800 $\mathrm{nm}$, with a repetition rate of $1 \mathrm{kHz}$ and a pulse duration of $100 \mathrm{fs}$. The laser beam was focused $150 \mu \mathrm{m}$ beneath of the surface by using a $20 \times$ microscope objective $(\mathrm{NA}=0.5)$. The $\mathrm{Nd}$ :YAB crystal was translated along its longest dimension (orthogonally to the propagation direction of the fs writing pulses) at a speed of $15 \mu \mathrm{m} / \mathrm{s}$. Optical WGs were fabricated following the so-called "double-line" design that requires the inscription of two parallel damage tracks. Several WGs were fabricated by using different separation distances and writing energies. We found that these parameters strongly determined the propagation properties of the resulting WGs. In 

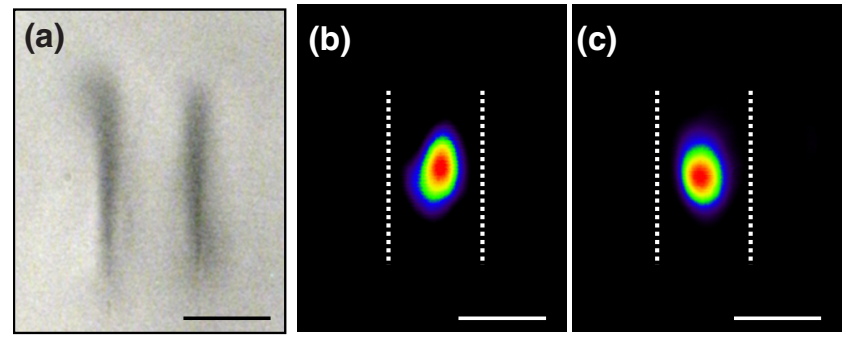

FIG. 1. (Color online) (a) Optical transmission cross-sectional image of the laser inscribed Nd:YAB WG fabricated with a pulse energy of $15 \mu \mathrm{J}$. (b) Near-field intensity distribution of a $1090 \mathrm{~nm}$ ordinary polarized mode. (c) Near-field intensity distribution of a $632.8 \mathrm{~nm}$ extraordinary polarized mode. Scale bar is $15 \mu \mathrm{m}$.

particular, the desired simultaneous TE and TM confinements for visible and infrared radiations were only obtained when the separation distance between damage tracks was reduced below $20 \mu \mathrm{m}$ and when (simultaneously) the writing energy was kept above $10 \mu \mathrm{J}$ per pulse. Best results were obtained when $15 \mu \mathrm{J}$ pulses were used to write $15 \mu \mathrm{m}$ separated filaments. The cross-sectional optical microscope image of this WG is shown in Fig. 1(a). Damage tracks were written parallel to the ordinary axis of the $\mathrm{Nd}$ :YAB sample. Figure 1(b) shows the near field distribution of the WG mode when an ordinary polarized $1090 \mathrm{~nm}$ laser was coupled into the WG. In addition Fig. 1(c) shows the near field distribution of the WG mode obtained when an extraordinary polarized visible $(632.8 \mathrm{~nm})$ laser beam was coupled into the WG.

It is clear from Fig. 1 that the fabricated WG supports both TM and TE polarizations in the visible-infrared spectral range. We state that the reduced separation between the two filaments, in addition to the large irradiation energies, have caused a large isotropic stress field between damage tracks that, due to piezo-optic effects, has caused a simultaneous increase of both ordinary and extraordinary refractive indices.

As previously stated in the introduction, the fabricated structures would be useful as SFD laser WGs only if the fluorescence and nonlinear properties of the active volume were not deteriorated during the laser inscription procedure. In order to check this, confocal imaging experiments were performed in a home-made fiber coupled confocal microscope that uses a mode-locked Ti:sapphire laser (Spectra Physics Tsunami) providing $100 \mathrm{fs}$ pulses at a repetition rate of $80 \mathrm{MHz}$. For fluorescence imaging experiments the Ti:sapphire laser was operated at $808 \mathrm{~nm}$ where $\mathrm{Nd}$ :YAB absorption is maximum. The $808 \mathrm{~nm}$ laser beam was focused onto the WG by using a $100 \times$ microscope objective $(0.9$ $\mathrm{NA}$ ). The back-scattered $\mathrm{Nd}^{3+}$ luminescence corresponding to the ${ }^{4} \mathrm{~F}_{3 / 2} \rightarrow{ }^{4} \mathrm{I}_{11 / 2}$ laser transition was collected by the same microscope objective and, after passing through several filters and confocal apertures, it was analyzed by a fibercoupled high resolution monochromator attached to a charge-coupled-device (CCD) camera. The sample was mounted on a high resolution XY motorized stage so that the fluorescence image of the WG's cross section was measured by scanning over it the excitation spot. For SH imaging the same setup was used but in this case the Ti:sapphire was tuned to $800 \mathrm{~nm}$ and only the $400 \mathrm{~nm}$ back-scattered SH signal was recorded.

Figure 2(a) shows the spatial variation of ${ }^{4} \mathrm{~F}_{3 / 2} \rightarrow{ }^{4} \mathrm{I}_{11 / 2}$ laser emission over the WG's cross section. As can be ob-

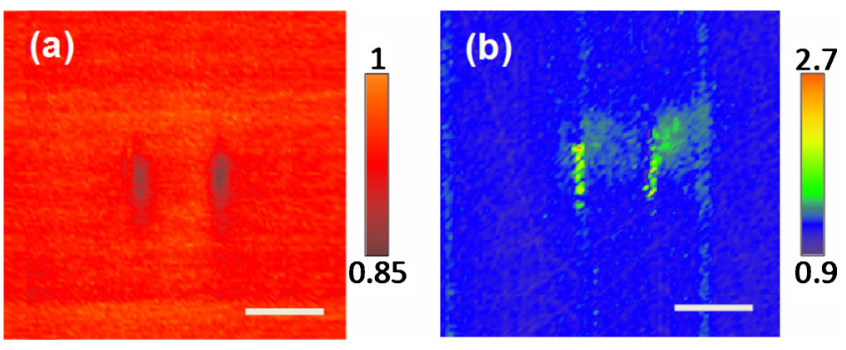

FIG. 2. (Color online) (a) Cross sectional confocal fluorescence image of the Nd:YAB WG as obtained from the spatial variation of the $\mathrm{Nd}^{3+}$ fluorescence intensity $\left({ }^{4} \mathrm{~F}_{3 / 2} \rightarrow{ }^{4} \mathrm{I}_{11 / 2}\right.$ laser channel). (b) Cross sectional SH image of the same Nd:YAB WG. Scale bar is $15 \mu \mathrm{m}$.

served the fluorescence efficiency of $\mathrm{Nd}^{3+}$ ions has been only partially deteriorated at damage filaments (probably due to the localized presence of lattice damage, as already stated in other "double-line" WGs) whereas at WG's core the fluorescence efficiency of $\mathrm{Nd}^{3+}$ ions remained unperturbed. Figure 2(b) shows the SH image of the WG's cross section, and it is clear that the nonlinear response (measured by the backscattering signal level) at the WG's volume has not been deteriorated in respect to the bulk one. This is, indeed, in accordance with previous works reporting on the nonlinear properties of ultrafast laser inscribed WGs in nonlinear crystals. ${ }^{8-11}$ The unexpected increment of the SH back intensity observed at damage tracks has been tentatively attributed (in accordance with previous works) to the presence in those volumes of a defect-assisted back-scattering enhancement. ${ }^{12}$ Thus, the confocal images of Fig. 2 unequivocally indicate that ultrafast laser inscription can be used to create optical WGs in YAB crystals without causing relevant degradation in its fluorescence and nonlinear optical properties.

The preservation of fluorescence and nonlinear properties at WGs volume, in combination with the ability of the WGs supporting TM and TE confinements in the green and infrared spectral ranges, make the fabricated structures outstanding candidates for the development of SFD WG lasers. In order to test this possibility, laser experiments were carried out. Two cavity mirrors were mechanically attached to the sample's $2 \mathrm{~mm} \times 2 \mathrm{~mm}$ faces by using an index matching gel $(\mathrm{n}=1.5)$. Input mirror was of high transmittance $(\approx 95 \%)$ at pump wavelength $(808 \mathrm{~nm})$ and of high reflectance $(\sim 99 \%)$ at laser wavelength of $1064 \mathrm{~nm}$. Output mirror was of high reflectance at pump $808 \mathrm{~nm}$ laser $(>99.8 \%)$ and $1064 \mathrm{~nm}$ laser $(\approx 97 \%)$, and of high transmittance $(>80 \%)$ at the SH wavelength $(532 \mathrm{~nm})$. Optical excitation of the channel WG was performed by end-fire coupling a Ti:sapphire continuous wave laser operating at 808 $\mathrm{nm}\left({ }^{4} \mathrm{I}_{9 / 2} \rightarrow{ }^{4} \mathrm{~F}_{5 / 2}\right.$ absorption band) into the WG by using a microscope objective. The geometry of the experiment was adopted to launch ordinary pump propagating modes into the channel WG in order to optimize the absorption and the spatial overlap with the desired ordinary polarized IR radiation. The laser emission from the WG was collected from the end face with a second microscope objective. For launched pump powers above $10 \mathrm{~mW}, 1064 \mathrm{~nm}$ ordinary polarized laser oscillation was achieved, being this accompanied by the generation of green radiation clearly observable by naked eye. The laser spectrum denoting the simultaneous presence of 1064 and $532 \mathrm{~nm}$ is shown in Figs. 3(a) and 3(b). The near field distributions of the visible and infrared laser modes are 

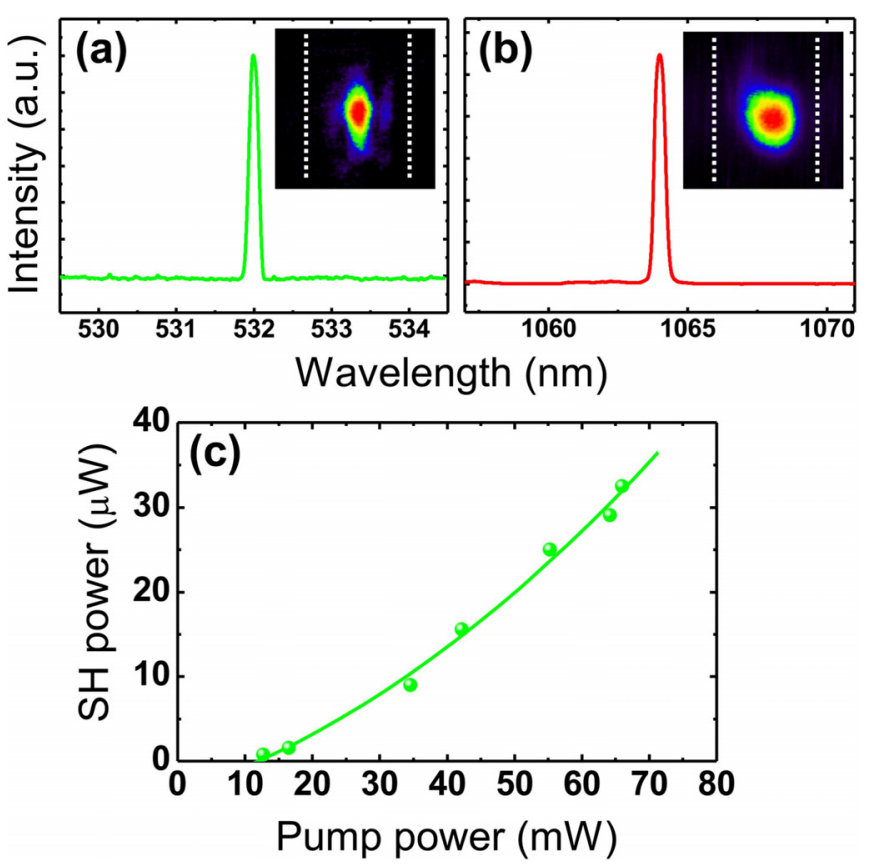

FIG. 3. (Color online) Spectral distribution of the (a) SH and (b) infrared laser radiations generated from the ultrafast laser inscribed Nd:YAB WG operating under $808 \mathrm{~nm}$ laser pumping. Insets show the corresponding near field distribution of SH and infrared laser. (c) Green power as a function of the pump power.

included in as insets. As can be observed, IR and visible radiations are oscillating in a $\mathrm{TEM}_{00}$ mode, this being an outstanding property since maximizes the spatial overlap between them (and enhance the nonlinear conversion efficiency).

Figure 3(c) shows the green laser power as a function of the $808 \mathrm{~nm}$ launched power as obtained from the WG of Fig. 1 . For the maximum launched power achievable in our experimental conditions $(70 \mathrm{~mW})$ we obtained up to $32 \mu \mathrm{W}$ of green laser radiation. For this $808 \mathrm{~nm}$ launched power we get up to $14 \mathrm{~mW}$ of fundamental radiation. The green output power level obtained in these experiments has been found to be significantly larger than those previously reported from the few SFD laser WGs demonstrated. Indeed, the maximum green power obtained from a SFD laser WG was $1.2 \mu \mathrm{W}$ from a quasi-phase-matching $\mathrm{Nd}: \mathrm{LiNbO}_{3} \mathrm{WG}$ laser. ${ }^{13} \mathrm{Nev}-$ ertheless, further optimization of the green output power is expected by, for example, using laser mirrors of higher reflectivity at $1064 \mathrm{~nm}$ (so that intracavity circulating powers would be reached) or by controlling sample temperature during laser experiments.

In summary, ultrafast laser inscription was used to fabricate buried channel optical WGs in Nd:YAB nonlinear laser crystal. An adequate choice of writing conditions leads to optical structures which support orthogonal polarization waveguiding in a wide spectral range without any deterioration neither in the fluorescence nor in the nonlinear properties of the bulk material. The ability of the fabricated WGs as green laser sources by SFD has been also demonstrated. Green output powers close to $40 \mu \mathrm{W}$ have been reported, beating previous performances of SFD WG lasers.

Work supported by the NSFC (Grant No. 10925524), the MICINN of Spain (Grant Nos. MAT 2010-16161, MAT2009-06580-E/MAT, FIS2009-09522, and CSD200700013), TE2010-Ginés, TEC2010-21574-C02-01, and the CAM and UAM of Madrid (Grant Nos. CCG08-UAM/MAT4434 and PHAMA P2009/MAT1756).

${ }^{1}$ A. Brenier, D. Jaque, and A. Majchrowskic, Opt. Mater. 28, 310 (2006).

${ }^{2}$ I. Schütz, I. Freitag, and R. Wallenstein, Opt. Commun. 77, 221 (1990).

${ }^{3}$ B. S. Lu, J. Wang, H. F. Pan, M. H. Jiang, E. Q. Liu, and X. Y. Hou, J. Appl. Phys. 66, 6052 (1989).

${ }^{4}$ D. Jaque, J. Capmany, J. García Solé, Z. D. Luo, and A. D. Jiang, J. Opt. Soc. Am. B 15, 1656 (1998).

${ }^{5}$ D. Jaque, J. Capmany, and J. García Solé, Appl. Phys. Lett. 75, 325 (1999).

${ }^{6}$ J. Bartschke, R. Knappe, K. J. Boller, and R. Wallenstein, IEEE J. Quantum Electron. 33, 2295 (1997).

${ }^{7}$ M. Q. Meng, F. Lu, K. M. Wang, F. X. Wang, W. Li, C. Q. Ma, D. H. Jiang, and Q. M. Lu, Electron. Lett. 33, 1045 (1997).

${ }^{8}$ B. McMillen, K. P. Chen, H. An, S. Fleming, V. Hartwell, and D. Snoke, Appl. Phys. Lett. 93, 111106 (2008).

${ }^{9}$ Y. Liao, J. Xu, Y. Cheng, Z. Zhou, F. He, H. Sun, J. Song, X. Wang, Z. Xu, K. Sugioka, and K. Midorikawa, Opt. Lett. 33, 2281 (2008).

${ }^{10}$ J. Thomas, M. Heinrich, P. Zeil, V. Hilbert, K. Rademaker, R. Riedel, S. Ringleb, C. Dubs, J. P. Ruske, S. Nolte, and A. Tünnermann, Phys. Stat. Solidi A 208, 276 (2011).

${ }^{11}$ R. Osellame, M. Lobino, N. Chiodo, M. Marangoni, G. Cerullo, R. Ramponi, H. T. Bookey, R. R. Thomson, N. D. Psaila, and A. K. Kar, Appl. Phys. Lett. 90, 241107 (2007).

${ }^{12}$ N. N. Dong, Y. Tan, A. Benayas, J. Vázquez de Aldana, D. Jaque, C. Romero, F. Chen, and Q. Lu, Opt. Lett. 36, 975 (2011).

${ }^{13}$ M. Fujimura, T. Kodama, T. Suhara, and H. Nishihara, IEEE Photonics Technol. Lett. 12, 1513 (2000). 